\title{
Misconceptions about Aerobic and Anaerobic Energy Expenditure
}

\author{
Christopher Scott \\ Sports Medicine Department, University of Southern Maine, Gorham, ME. Address correspondence to \\ cscott@usm.maine.edu
}

Received October 10, 2005/Accepted November 2, 2005

\begin{abstract}
The measurement of gas exchange has played an invaluable role in metabolic interpretation. The uptake of 1 liter of oxygen is often converted into an energy expenditure estimate of 21.1 kilojoules (e.g., $1 \mathrm{~L} \mathrm{O}_{2}=21.1$ $\mathrm{kJ}$ or $\sim 5 \mathrm{kcal})$. This article demonstrates both the importance of such a conversion and the potential for misinterpretation. Oxygen uptake during heavy and severe exercise will also be discussed. Journal of the International Society of Sports Nutrition. 2(2):32-37, 2005
\end{abstract}

\section{INTRODUCTION}

The measurement of gas exchange - as oxygen $\left(\mathrm{O}_{2}\right)$ uptake and carbon dioxide $\left(\mathrm{CO}_{2}\right)$ production - has played an invaluable role in metabolic interpretation where, for example, 1 liter of $\mathrm{O}_{2}$ uptake can be converted into an energy expenditure estimate of 21.1 kilojoules (e.g., $1 \mathrm{~L} \mathrm{O}_{2}=21.1 \mathrm{~kJ}$ or $\left.\sim 5 \mathrm{kcal}\right)$. This manuscript demonstrates both the importance of such a conversion and the potential for misinterpretation. Heat represents the standard measure of energy expenditure. Oxygen uptake estimates metabolic heat production ${ }^{1}$. It is well documented that $\mathrm{O}_{2}$ uptake measurements are often but not always proportional to metabolic heat production. Short-lived, heavy to severe exercise serves as one example. $\mathrm{O}_{2}$ uptake during brief and intense exercise and the recovery from that exercise (i.e., the $\mathrm{O}_{2}$ debt) does not represent every component of energy expenditure as was once proposed and is still widely practiced. Now-a-days the term ' $\mathrm{O}_{2}$ debt' has been changed to excess post-exercise $\mathrm{O}_{2}$ consumption (EPOC) but this does nothing in terms of quantifying anaerobic energy expenditure. While it is certainly true that measurements of anaerobic energy expenditure are questionable, reasonable estimates of rapid glycolytic ATP turnover are available and should not be omitted from the estimation of energy expenditure, especially when the anaerobic contributions are large.

\section{ENERGY EXPENDITURE DESCRIBES ENERGY TRANSFER}

The study of energy expenditure begins with observations that have been made in regard to energy transfer. 'Energy' is defined as the ability to do work or the capacity to cause change. The term 'transfer' denotes a removal or shifting from one place to another. Energy expenditure is a measure that informs us how much energy is required to perform a given work bout or work rate (quantitative in Joules; as a rate in Watts). Some of the observations regarding energy transfer were made centuries ago and because they have not been disproved they are regarded as laws. The $1^{\text {st }}$ law describes energy itself and states that energy is conserved, it is not created nor can it be destroyed. The $2^{\text {nd }}$ laws describe the transfer of energy from one form to another where it has been recognized that spontaneous transfer always occurs in the "downhill" direction (e.g., heat flows toward cold, never the opposite). Another of the $2^{\text {nd }}$ laws states that while energy can be transferred from one form to another the exchange is never perfect; energy transfer is inefficient. Efficiency is defined as the amount of work completed divided by the amount of energy required to perform that work (the value is provided as a percentage). The most readily recognizable component of energy transfer is heat production. It is with this knowledge that the measurement of heat serves as the standard means to interpret both energy transfer efficiency and energy expenditure. 


\section{ENERGY EXPENDITURE, HEAT PRODUCTION AND GAS EXCHANGE}

It was recognized over 2,000 years ago that heat and life were related ${ }^{2}$. This no doubt set the stage for the measurement of heat production as a way to quantify biological energy expenditure. Slightly over 200 years ago it was recognized that biological respiration and combustion gave off similar amounts of heat and $\mathrm{CO}_{2}{ }^{3,4}$. Soon afterward it became understood that both respiration and combustion were processes that also took $\mathrm{O}_{2}$ from the air so that heat production and gas exchange $\left(\mathrm{O}_{2}\right.$ uptake and $\mathrm{CO}_{2}$ production) were related ${ }^{4}$.

The next breakthrough was of a mechanical origin (not biological) and came from mid- $19^{\text {th }}$ century scholars (physicists) who studied steam engines. The 'laws' that resulted from this inquiry defined the limitations behind heat-to-mechanical energy transfer; this area of study is known as thermodynamics. Of course biological energy transfer has boundaries too and this is studied as bioenergetics ${ }^{5}$. Thermodynamics has a heavy influence on bioenergetics but the two are not exactly alike (see Bioenergetics below). It is therefore ironic that while early biochemists came to understand that muscle was a chemical-to-mechanical motion converter (not a heat-to-mechanical motion converter like the steam engine), it was thermodynamics rather than bioenergetics that was initially applied to interpretations of aerobic and anaerobic energy transfer.

At the start of the $20^{\text {th }}$ century heat and $\mathrm{O}_{2}$ uptake measurements were determined to be proportional in normal, resting, adult, human subjects residing in a temperate environment ${ }^{6}$. Measuring the heat loss of human subjects, a process known as direct calorimetry, has been and continues to be a technically difficult and labor intensive endeavor. Fortunately, tremendous advancements have been made in those devices that measure gas exchange, a process known as indirect calorimetry. The measurement of $\mathrm{O}_{2}$ uptake in laboratory, hospital and academic settings world-wide has, over time, led many to construe $\mathrm{O}_{2}$ uptake and energy expenditure as one and the same. In fact, they often are. Yet it must be kept soundly in mind that heat measurement serves as the gold standard in the interpretation of energy transfer efficiency and energy expenditure; $\mathrm{O}_{2}$ uptake is used to estimate heat production ${ }^{7}$. This needs to be well understood because while both anaerobic and aerobic energy expenditure results in heat production, only the latter results in $\mathrm{O}_{2}$ uptake.

\section{BIOENERGETICS}

Exercising muscle, like all cells, requires energy to function. That energy is found within the molecular bonds of adenosine tri-phosphate (ATP). When the chemical energy within ATP is transferred to muscle contraction, adenosine di-phosphate (ADP) and phosphate $(\mathrm{Pi})$ are formed (ATP $\rightarrow$ ADP $+\mathrm{Pi}$ ). Additional energy is required to reverse this reaction $(\mathrm{ATP} \leftarrow \mathrm{ADP}+\mathrm{P})$. The energy required to resynthesize ATP is transferred from the food we eat carbohydrates and fats - via specific biochemical pathways within our cells. The cycle of ATP hydrolysis via muscle contraction and ATP resynthesis via aerobic and anaerobic metabolic biochemistry is know as ATP turnover. Bioenergetic descriptions of ATP turnover reveals that while ATP is re-synthesized from $\mathrm{ADP}$ and $\mathrm{Pi}$, it is not a thermodynamically reversible reaction; heat production (energy expenditure) results from those events associated with both ATP hydrolysis and resynthesis.

Anaerobic and aerobic metabolisms each utilize distinct energy transfer mechanisms even though both serve to re-synthesize ATP. They each take place in separate cellular compartments and often at different rates, involve different reactants, products and product/reactant ratios, invoke unlike types of biochemical reactions, exploit different types of energy gradients and subsequently appear to operate with distinct efficiency; these conditions further distinguish bioenergetics from thermodynamics ${ }^{8}$.

\section{ANAEROBIC METABOLISM}

The breakdown of carbohydrates - glucose and glycogen - involves both anaerobic and aerobic biochemistry. Energy is contained within the molecular bonds of glucose and is released during their breakdown in a process called glycolysis (glycogen is the storage form of glucose and is broken down as glycogenolysis). Glycolysis starts with a 6-carbon sugar as a reactant and ends up with two 3-carbon molecules as products. Glycolysis also re-synthesizes ATP, a process termed substrate level phosphorylation. This type of substrate level phosphorylation takes place in the cytoplasm of cells, within and around the contractile apparatus of muscles for example. These reactions can be generalized as a series of phosphate transfers, phosphate shifts, dehydrations and molecular cleavage. The reactions proceed "downhill" because the useful energy found within the metabolic products (e.g., lactate) is less than the energy found 
within the reactant (e.g., glucose). Glycolysis invokes proton $\left(\mathrm{H}^{+}\right)$, electron $(e-)$, and phosphate $(\mathrm{Pi})$ transfer along the way from reactants to products.

There can actually be two finales to glycolytic substrate level phosphorylation. When the anaerobic removal of $\mathrm{H}^{+}$and $e$ - from glucose or glycogen is matched to the rate of aerobic metabolism, then pyruvate is the end product. Sometimes however, as with heavy to severe exercise, rapid substrate level phosphorylation proceeds at a rate that exceeds aerobic metabolism ${ }^{9}$. Instead of being carried toward the site of aerobic metabolism, the rapidly removed $\mathrm{H}^{+}$and $e$ - are accepted by pyruvate to become lactate. Lactate appearance is a natural part of an accelerated anaerobic metabolism where substrate level phosphorylation provides a significant amount of ATP and heat (energy expenditure). The more intense the exercise the faster is the rate of ATP hydrolysis and the more glucose and glycogen are the preferred fuel to re-synthesize ATP.

Lactate disappearance occurs through a variety of means. The pyruvate to lactate reaction is reversible (but the heat produced from the rapid formation of lactate is not ${ }^{10}$ ). Pyruvate may then undergo subsequent aerobic metabolism (see below). Or, lactate can go to the liver and be re-converted back to glucose, or glycogen within muscle, or alanine (an amino acid). These conversions require energy that is supplied by aerobic metabolism.

\section{AEROBIC METABOLISM}

Fat is the preferred fuel at rest and during lower intensity exercise; energy transfer from fat occurs only via aerobic biochemistry. Energy expenditure during the aerobic re-synthesis of ATP is not related to anaerobic substrate level phosphorylation. Instead, aerobic ATP re-synthesis takes place within a specific cellular organelle known as the mitochondria. Mitochondria consist of two doublemembranes that effectively separate the inner mitochondrial compartment from the cells cytoplasm. Mitochondria continue to removed $\mathrm{H}^{+}$and $e$ - from pyruvate or fatty acids via a metabolic pathway known as the Kreb's cycle. Oxygen attracts $e$ - as part of the electron transport chain that is located within the inner mitochondrial membrane. Using the inner membrane as a barrier, $\mathrm{H}^{+}$that are stripped from pyruvate and fatty acids are pumped from one side of the membrane to the other; this pumping is fueled by the electron transport chain. The subsequent uphill (high $\mathrm{H}^{+}$concentration) to downhill (low $\mathrm{H}^{+}$ concentration) scenario creates an electro-chemical energy gradient. Specific protein portals within the inner mitochondrial membrane called ATPases allow the passage of $\mathrm{H}^{+}$down its gradient and the energy of this flow is transferred to ATP re-synthesis. The creation and exploitation of $\mathrm{H}^{+}$and $e$ - gradients via the use of membranes is termed respiration. Much of aerobic heat production has been found to result from the flow of $\mathrm{H}^{+}$down its respective gradient ${ }^{11}$.

\section{$\mathrm{O}_{2}$ UPTAKE AND ENERGY EXPENDITURE}

The use of $\mathrm{O}_{2}$ uptake measurements to interpret biological efficiency and energy expenditure was well received and continues to undergo widespread promotion. Indeed, the word 'oxygen' has been applied to nearly all phases of energy transfer whether aerobic or anaerobic. At the start of exercise for example, a brief period exists that is associated with anaerobic energy expenditure; this was described as the oxygen deficit ${ }^{12}$. Moreover, the quantification of all anaerobic energy expenditure was said to be founded in the oxygen debt after exercise had ended ${ }^{13}$. In fact, anaerobic and aerobic metabolism are often combined into a single interpretation of energy expenditure where 1 liter $\mathrm{O}_{2}$ $=21.1 \mathrm{~kJ}$ or $\sim 5 \mathrm{kcals}$. This conversion is meaningful but also has the potential for misinterpretation.

In 1917 William Thornton revealed the strikingly linear relationship between the quantity of $\mathrm{O}_{2}$ consumed and how much heat is produced during combustion ${ }^{14}$. "...when combustion is complete with one oxygen atom the heat set free, irrespective of bonds, is 52.4 calories per gram molecule of combustible gas, when with two atoms 105.9, with three $158.59^{14}$." This relationship is similar for metabolic respiration ${ }^{15}$. Thus, the sole measurement of $\mathrm{O}_{2}$ uptake does not make any energetic distinction of $e$ - transfer between glucose and fat to molecular $\mathrm{O}_{2}$ (energy transfer per $\mathrm{O}_{2}$ equivalent is the same regardless of the fuel; that is Thornton's law). Yet nutrition and exercise physiology texts usually focus on what are very different conversions. For example, the $\mathrm{O}_{2}$ uptake of glucose oxidation has been converted into an estimate of heat production as 1 liter $\mathrm{O}_{2}=21.1 \mathrm{~kJ}$; fat oxidation is estimated at 1 liter $\mathrm{O}_{2}=19.6 \mathrm{~kJ}$. Per liter of $\mathrm{O}_{2}$ uptake then, there is a 1.5 $\mathrm{kJ} \quad(\sim 7 \%)$ difference between glucose and fat metabolic heat production.

How is it that the metabolic energy expenditure during glucose and fat metabolism is in apparent contradiction of Thornton's finding? The conversion of $\mathrm{O}_{2}$ uptake into an estimate of metabolic energy expenditure is ultimately an attempt to quantify ATP 
turnover per $\mathrm{O}_{2}$ equivalent. As mentioned earlier both anaerobic and aerobic energy expenditure results in heat production, but only the latter results in $\mathrm{O}_{2}$ uptake. It also is important to recognize that the type of substrate being oxidized (glucose or fat) must be known before an $\mathrm{O}_{2}$ uptake to heat conversion can take place and that requires a measurement of $\mathrm{CO}_{2}$ (the ratio of $\mathrm{CO}_{2} / \mathrm{O}_{2}$ determines substrate utilization as the respiratory exchange ratio). Using the typical example of 1 molecule of glucose, it is known that $\sim 36$ ATP are re-synthesized as anaerobic and aerobic oxidation take place. Two ATP are from anaerobic glycolytic substrate level phosphorylation representing $\sim 6 \%$ of total ATP re-synthesis per glucose related $\mathrm{O}_{2}$ equivalent (at $1.5 \mathrm{~kJ}$ per liter of $\mathrm{O}_{2}$ ); the other $\sim 94 \%$ of ATP are re-synthesized aerobically (at $19.6 \mathrm{~kJ}$ per liter of $\mathrm{O}_{2}$ ). The $6 \%$ increase in glycolytic ATP formation is directly related to the $7 \%$ increase in heat production because - as the $2^{\text {nd }}$ law dictates - all energy transfer is inefficient. The linkage of fat oxidation with ATP resynthesis is $100 \%$ aerobic, has no anaerobic component and thus has slightly less heat production per $\mathrm{O}_{2}$ uptake equivalent (at $19.6 \mathrm{~kJ}$ per liter of $\mathrm{O}_{2}$ ). Heat production that exceeds $19.6 \mathrm{~kJ}$ per liter of $\mathrm{O}_{2}$ has been clearly shown to contain an anaerobic glycolytic component ${ }^{15}$.

\section{MEASUREMENT VALIDITY}

The measurement of $\mathrm{O}_{2}$ uptake to quantify energy expenditure does not pose validity problems when the substrate being oxidized is known (glucose or fat), when using precision equipment that is regularly calibrated and when the measurements are taken using 'normal' subjects at rest or undergoing light to moderate steady-rate exercise. During brief heavy to severe exercise $\mathrm{O}_{2}$ uptake does not spontaneously arise to meet metabolic demands (this is studied as $\mathrm{O}_{2}$ uptake kinetics). When rapid glycolysis with lactate production makes a significant contribution to total ATP turnover, then an anaerobic measurement also is required to account for this type of energy transfer. Thus, exercise $\mathrm{O}_{2}$ uptake is an invalid measure of energy expenditure for brief intense non-steady-rate exercise that has a large glycolytic ATP turnover component (see table 1).

In similar fashion to aerobic energy expenditure an acceptable measure of anaerobic energy expenditure would appear to reside in the collection of a representative anaerobic metabolite. And therein lays the problem. It is very difficult to obtain biochemical samples from within active skeletal muscle cells, especially when it is seldom known how much muscle and what type of muscle is being recruited. Lactate is one readily recognized example. Different types of skeletal muscles (fast and slow twitch) produce differing amounts of lactate and the turnover of this metabolite is extensive (fast twitch muscle is a lactate producer; slow twitch muscle is a lactate consumer). Moreover, muscle and blood lactate concentrations can be very different. It is much easier to obtain a blood lactate as opposed to a muscle lactate sample. Yet because of the aforementioned difficulties, blood lactate concentrations have been justifiably criticized as an invalid means to quantify anaerobic energy expenditure. Even so, attempts have been made by scientists over several decades that have provided some indication of the extent of rapid anaerobic glycolytic ATP turnover using blood lactate measurements ${ }^{16,17}$.

Problems with the interpretation of energy expenditure for brief intense non-steady-rate exercise are readily apparent. But it must also be kept in mind that researchers who view all anaerobic measurements as invalid and unacceptable have a double standard if they also accept invalid $\mathrm{O}_{2}$-only measurements as the sole means of quantifying energy expenditure for exercise that has a large glycolytic ATP turnover component.

\section{INCORRECT MEASUREMENT, INCOMPLETE MEASUREMENT AND REASONABLE ESTIMATE}

Years ago exercise physiologists were taught that rapid glycolytic ATP turnover (i.e., anaerobic substrate level phosphorylation) could be quantified by measuring the $\mathrm{O}_{2}$ uptake during the recovery from exercise. This was known as the $\mathrm{O}_{2}$ debt hypothesis. It is now clear that the $\mathrm{O}_{2}$ debt represents an incorrect measurement of rapid glycolytic ATP turnover ${ }^{10,18}$. The term $\mathrm{O}_{2}$ debt has been replaced with excess postexercise $\mathrm{O}_{2}$ consumption or EPOC ${ }^{18}$. Glycolytic ATP turnover can be altogether dismissed from the EPOC measurement by using the conversion of 1 liter of $\mathrm{EPOC}=19.6 \mathrm{~kJ}{ }^{8}$. Unfortunately, changing the name of the $\mathrm{O}_{2}$ debt to EPOC and/or dismissing the glycolytic ATP turnover component from an $\mathrm{O}_{2}$ uptake measurement does nothing to quantify anaerobic energy expenditure. As exercise intensity increases both the anaerobic and EPOC components can increase significantly so that the omission of either these components results in an incomplete measurement of total energy expenditure. There are 
Table 1. Aerobic and anaerobic energy expenditure for 2 exercise tasks of equal work

\begin{tabular}{|c|c|c|}
\hline Measure & $\underline{\text { Steady rate walk }}$ & Intermittent sprints \\
\hline Exercise $\mathrm{O}_{2}$ & $120 \mathrm{~kJ}$ & $16 \mathrm{~kJ} *$ \\
\hline $\begin{array}{l}\text { Exercise } \mathrm{O}_{2} \\
+ \text { EPOC }\end{array}$ & $149 \mathrm{~kJ}$ & $165 \mathrm{~kJ}$ \\
\hline $\begin{array}{l}\text { Anaerobic + } \\
\text { Exercise } \mathrm{O}_{2} \\
+ \text { EPOC }\end{array}$ & $164 \mathrm{~kJ}$ & $273 \mathrm{~kJ} *$ \\
\hline \multicolumn{3}{|c|}{$\begin{array}{l}\text { Each of the tasks involved } 27.1 \pm 2.3 \mathrm{~kJ} \text { of treadmill work. Anaerobic energy expenditure was measured as the } \mathrm{O}_{2} \text { deficit. Steady rate } \\
\text { exercise required } 3.5 \text { minutes and was performed at } 47 \% \text { of } \mathrm{VO}_{2} \text { max. Intermittent exercise was completed as three } 15 \text {-sec sprints at } \\
\sim 177 \% \text { of } \mathrm{VO}_{2} \text { max. The * indicates a statistical difference from the steady rate value. Both the significant and non-significant findings } \\
\text { have importance. For example, without a reasonable estimate of anaerobic energy expenditure it may be falsely concluded that the } \\
\text { energy expenditure (i.e., exercise } \mathrm{O}_{2} \text { uptake }+ \text { EPOC) for these two walking and sprinting tasks was similar }(149 \mathrm{vs} 165 \mathrm{~kJ} \text {, respectively) } \\
\left.\text { when they are significantly different ( } 164 \mathrm{vs} 273 \mathrm{~kJ} \text {, respectively) (adapted from }{ }^{26}\right) \text {. The estimate of anaerobic energy expenditure also } \\
\text { reveals that this component is not significant for the low intensity steady rate walk ( } 149 \mathrm{vs} 164 \mathrm{~kJ} \text { ) but it is for the intense intermittent } \\
\text { sprints }(165 \mathrm{vs} 273 \mathrm{~kJ}) \text {. Based on exercise } \mathrm{O}_{2} \text { uptake between the steady rate walk and intense intermittent sprints }(120 \mathrm{vs} 16 \mathrm{~kJ} \text {, } \\
\text { respectively) it was concluded that } \mathrm{O}_{2} \text { uptake is an invalid measure of exercise energy expenditure for brief intermittent severe exercise }\end{array}$} \\
\hline
\end{tabular}

times when an incomplete measurement is no better than an incorrect measurement.

As mentioned previously, blood lactate measurements have provided a reasonable estimate of anaerobic glycolytic ATP turnover and this may be of more help than hindrance in the quantification of total energy expenditure. To illustrate this point it must be acknowledged that all branches of science (and nonscientists too for that matter) have found use for indirect measures or estimates when direct measures are lacking. Because the contribution of anaerobic energy expenditure to brief heavy to severe exercise can be significant, the greatest error in quantifying total energy expenditure for intense exercise may occur not by using a reasonable estimate of anaerobic energy expenditure but instead by omitting all estimates of anaerobic energy expenditure ${ }^{19}$. Two examples from our lab describe the potential for misinterpretation when reasonable estimates of anaerobic energy expenditure are ignored. First, $\mathrm{O}_{2}$-only measurements of circuit weight training have led to the conclusion that certain formats of this type of exercise may not provide enough energy expenditure to derive health benefits 20; but when we added in the anaerobic energy expenditure component, we found that many formats of circuit weight training do indeed expend enough energy to promote health ${ }^{21}$ (see Table 1 for a comparison between walking and sprinting). Secondly, previous comparisons between cycling and running have attempted to equate these two tasks based on self-selected exercise intensity ${ }^{22}$, perceived exertion ${ }^{23}$, and as a percentage of maximum HR ${ }^{24}$ and $\mathrm{O}_{2}$ uptake ${ }^{23}$. These studies all found that cycling results in a lower energy expenditure (i.e., $\mathrm{O}_{2}$ uptake) and a greater lactate production as compared to running. When we equated work between brief bouts of cycling and running we found similar results. When we converted blood lactate into a reasonable estimate of anaerobic energy expenditure and added it to the aerobic energy expenditure $\left(\mathrm{O}_{2}\right.$ uptake) we found that energy expenditure for both tasks was identical $^{25}$.

\section{SYNOPSIS}

$\mathrm{O}_{2}$ uptake and metabolic heat production are often proportional and the demonstration of this was a tremendous scientific achievement. This proportionality however is only found in adult, nondiseased subjects residing in a temperate environment, and those resting or engaged in light to moderate exercise. It must be noted that under certain conditions metabolic heat production can exceed $\mathrm{O}_{2}$ uptake. The potential error of using an $\mathrm{O}_{2}-$ only measurement to interpret the energy expenditure of heavy to severe exercise justifies the need for and use of a reasonable estimate of anaerobic energy expenditure to better quantify total energy expenditure. In the mean time, research must continue on finding a universally accepted standard for the determination of anaerobic energy expenditure. 


\section{REFERENCES}

1. Zotin, A.I. Thermodynamic Bases of Biological Processes. Walter de Gruyter, Berlin. 1990.

2. Mendelsohn, E. Heat and Life: The Development of the Theory of Animal Heat. Harvard University Press: Cambridge, 1964.

3. Blaxter, K.L. Symposium on the application of human and animal calorimetry. Proc. Nutr. Soc. 37:1-3, 1978.

4. Donovan, A. Antoine Lavoisier: Science, Administration and Revolution. Blackwell Publ.: Oxford, 1993.

5. Nicholls, D.G. and S.J. Ferguson. Bioenergetics 3. Academic Press: Amsterdam, 2002.

6. Benedict, F.G. and R.D. Milner. Experiments on the metabolism of matter and energy in the human body, 1903-1904. U.S. Department of Agriculture, Office of Experiment Station - Bulletin 175. Washington: Government Printing Office, 1907.

7. Kleiber, M. The Fire of Life: An Introduction to Animal Energetics. Robert E. Krieger Publ.: Malabar, FL, 1987.

8. Scott, C.B. Contributions of anaerobic energy expenditure to whole-body thermogenesis. Nutr. Metab. 2:14, 2005.

9. Spriet, L.L. Anaerobic metabolism during high-intensity exercise. In, Exercise Metabolism, M. Hargreaves, Ed., Human Kinetics Publ.: Champaign, Il, 1995.

10. Scott, C.B. and Kemp, R.B. Direct and indirect calorimetry of lactate oxidation: implications for whole-body energy expenditure. J. Sports Sci. 23:15-19.

11. Brand, M.D., et al. The causes and functions of mitochondrial proton leak. Biochim Biophys Acta. 1187:132-139, 1994.

12. Krogh, A. and J. Lindhard. The changes in respiration at the transition from work to rest. J Physiol. 53:431-439, $1919 / 20$.

13. Hill, A.V., C.N.H. Long and H. Lupton. Muscular exercise, lactic acid and the supply and utilization of oxygen. Proc. Roy. Soc. B. 96:438-475, 1924.

14. Thornton, W.M. The relation of oxygen to the heat of combustion of organic compounds. Philos. Mag. Ser. 33:196-203, 1917.

15. Gnaiger, E. and R.B. Kemp. Anaerobic metabolism in aerobic mammalian cells: information from the ratio of calorimetric heat flux and respirometric oxygen flux. Biochim. Biophys. Acta. 1016:328-332, 1990.

16. di Prampero, P.E. and Ferretti, G. The energetics of anaerobic muscle metabolism: a reappraisal of older and recent concepts. Resp. Physiol. 118:103-115, 1999.

17. Margaria, R., Aghemo, P. and Sassi, G. Lactic acid production in supramaximal exercise. Pflug. Arch. 326:152-161, 1971.

18. Gaesser, G.A. and Brooks, G.A. Metabolic bases of excess post-exercise oxygen consumption: a review. Med. Sci. Sports Exer. 16:29-43, 1984.

19. Scott, C.B. Estimating energy expenditure for brief bouts of exercise with acute recovery. Can. J. Appl. Physiol. (in press).

20. Phillips, W.T. and Ziuraitis, J.R. Energy cost of the ACSM single-set resistance training protocol. J. Strength Cond. Res. 17:350-355, 2003.

21. Scott, C.B. Contribution of blood lactate to the energy expenditure of weight training. J. Strength Cond. Res. (in press).

22. Kravitz, L. et al. Exercise mode and gender comparisons of energy expenditure at self-selected exercise intensities. Med Sci Sports Exerc. 29:1028-1035, 1997.

23. Thomas, T.R. et al. Physiological and perceived exertion responses to six modes of submaximal exercise. Res Q Exerc Sport. 66:239-246, 1995.

24. Thomas, T.R. et al. Metabolic responses associated with four modes of prolonged exercise. J Sport Med. 29:77-82, 1989.

25. Scott, C.B., et al. Differences in VO2 and lactate but similarity in energy expenditure between equivalent bouts of cycling and running. (in review).

26. Scott, C.B. Interpreting energy expenditure for anaerobic exercise and recovery: an anaerobic hypothesis. J Sports Med Phys Fit. 37:18-23, 1997. 